\title{
ANÁLISIS DEL PEDIGRÍ Y EFECTOS DE LA CONSANGUINIDAD EN EL COMPORTAMIENTO DEL GANADO DE LIDIA MEXICANO
}

\author{
PEDIGREE ANALYSIS AND EFFECTS OF INBREEDING BEHAVIOR \\ OF MEXICAN FIGHTING BULL
}

\begin{abstract}
Domínguez Viveros, J. ${ }^{1}$, Rodríguez Almeida, F.A. ${ }^{1}$, Núñez Domínguez, R. ${ }^{2}$, Ramírez Valverde, R. ${ }^{2}$, Ortega Gutierrez, J.A. ${ }^{1}$ y Ruíz Flores, A. ${ }^{2}$
\end{abstract}

\begin{abstract}
${ }^{1}$ Facultad de Zootecnia y Ecología. Universidad Autónoma de Chihuahua. Periférico Francisco R. Almada km 1. CP 31031. Chihuahua, Chihuahua. México. jodominguez@uach.mx

${ }^{2}$ Departamento de Zootecnia. Universidad Autónoma Chapingo. Carretera México-Texcoco km 38.5. CP 56230. Texcoco, Estado de México. México.rafael.nunez@correo.chapingo.mx
\end{abstract}

\section{Palabras ClaVe adicionales}

Toros de Lidia. Ancestros. Fundadores. Intervalo generacional. Endogamia.

\section{RESUMEN}

El objetivo fue analizar el pedigrí de cuatro ganaderías mexicanas (Los Encinos=ENC, Montecristo=MCR, Fernando de la Mora=FMO y San José=SJO) a través de estimaciones de parámetros poblacionales tales como el tamaño de población base, tamaño efectivo, número de ancestros, aportaciones porcentuales e intervalos generacionales. Además se analizó el efecto de la consanguinidad sobre el comportamiento de los animales (tienta y lidia al caballo y al torero) con un modelo animal que consideró como efectos fijos a grupo contemporáneo (año-época de nacimiento-sexo, el efecto de sexo sólo en tienta) y las covariables lineales de edad y consanguinidad del individuo; los efectos aleatorios fueron el valor genético del individuo y el error. Los pedigríes incluyeron de 3246 a 8279 animales nacidos entre 1904 y 2006; la información de comportamiento data de 1978 a 2006, variando de 202 a 2776 observaciones. Las consanguinidades promedio variaron entre $2,4 \%$ y $12,9 \%$, con máximos individuales de $47,7 \%$ en MCR y de alrededor de $40 \%$ en las otras ganaderías. El número de ancestros como proporción de la población de referencia en MCR fue $12,3 \%$, mientras que en FMO, ENC y SJO representaron $17,7 \%, 27,8 \%$ y $34,0 \%$, respectivamente. Tres animales explicaron $50 \%$ del pedigrí en MCR y 13 o menos en las otras ganaderías. Los tres ancestros de MCR explicaron 22,6\% y

Recibido: 22-10-07. Aceptado: 14-5-08.

\section{AdDitional KEYWORDS}

Bullfighting. Ancestors. Founders. Generation interval. Inbreeding.

$20,2 \%$ del pedigrí de SJO y ENC, respectivamente. Las ganaderías MCR, SJO y ENC tienen un origen común. Las tendencias anuales de consanguinidad fueron negativas en ENC y SJO $(-0,13 \pm 0,02$ y $-0,25 \pm 0,02$, respectivamente; $p<0,01)$; mientras que las tendencias de la proporción de animales consanguíneos fueron positivas en FMO y MCR $(2,41 \pm 0,41$ y $0,16 \pm 0,05$, respectivamente; $p<0,01)$. La consanguinidad se relacionó significativamente $(p<0,05)$ sobre las notas de tienta en ENC y SJO, y lidia al torero en SJO. La calificación en las notas aumentó conforme los niveles de consanguinidad del animal fueron mayores. La magnitud de los coeficientes de regresión varió entre 0,02 y 0,05 unidades en las notas por unidad porcentual de la consanguinidad.

\section{SUMMARY}

The objective was to analyze the pedigree of four Mexican ranches (Los Encinos=ENC, Montecristo $=M C R$, Fernando de la Mora $=F M O$, and San José=SJO) through estimates of base population, effective size, reference population, number of ancestors, percentage of contribution, and generation intervals. Also, the effect of inbreeding on the animals behavior (testing young animals and fighting on horseback or by bullfighter) was analyzed with an animal model that considered 
as fixed effects contemporary group (yearseason of birth-sex, the sex effect only in testing young bulls) and the linear covariables of age and inbreeding of the animal. Random effects were the breeding value of the animal and the residual. The number of animals in the pedigree varied from 3246 to 8279 born between 1904 and 2006; the behavior records were from 1978 to 2006, ranging from 202 to 2776 . Average inbreeding levels varied from 2.4 to $12.9 \%$, with a maximum individual value of $47.7 \%$ in MCR and around $40 \%$ in the other ranches. The number of ancestors as a proportion of the reference population on MCR was $12.3 \%$, while in FMO, ENC, and SJO represented 17.7\%, 27.8\% and $34.0 \%$, respectively. Three ancestors explained $50 \%$ of the pedigree in MCR and 13 or less in the other ranches. The three ancestors in MCR explained $22.6 \%$ and $20.2 \%$ of the pedigree in SJO and ENC, respectively. The MCR, SJO, and ENC ranches had a common origin in their pedigrees. The annual inbreeding trends were negative in ENC and SJO $(-0.13 \pm 0.02$ and $-0.25 \pm 0.02$, respectively; $p<0.01$ ); while the trends in the proportion of inbred animals were positive in $\mathrm{FMO}$ and MCR (2.41 \pm 0.41 and $0.16 \pm 0.05$, respectively; $p<0.01)$. The ingreeding had a significant effect $(p<0.05)$ on testing scores of young animal in ENC and SJO, and on bullfighthing scores in SJO. The score of testing young animals and bullfigthing increased as the level of inbreeding of the animal augmented. The size of the regression coefficient varied from 0.02 and 0.05 units of the trait per unit of percentage of inbreeding.

\section{INTRODUCCIÓN}

La ganadería de toros de Lidia se ha desarrollado en la mayor parte de la República Mexicana. La actividad se practica bajo condiciones extensivas, con un mínimo de manejo y evitando el contacto con el hombre, por lo que esta raza como recurso genético puede contener genes únicos de adaptación y rusticidad. El origen de las actuales ganaderías de Lidia mexicanas se dio en unas 16 explotaciones de cuatro familias de criadores. El antecedente más antiguo, y que aún persiste en la actualidad, es la ganadería Atenco en el estado de México, cuya explotación se remonta al año 1522 (Lanfranchi, 1992; Castillo, 2003).
El estudio de la constitución genética de las poblaciones y su evolución a través de las generaciones, es la base para el establecimiento y desarrollo de programas de selección o de conservación. El análisis de los datos del pedigrí es una herramienta importante para describir la constitución genética de las poblaciones. Los principales indicadores que se utilizan en el estudio de los pedigríes son: tamaño efectivo de población, número efectivo de ancestros y aportaciones porcentuales de los ancestros fundadores (Boichard et al., 1997; Parland et al., 2007), estructura de hatos, ganaderías o subpoblaciones y sus contribuciones con reproductores (Vasallo et al., 1986; Gutiérrez et al., 2003), intervalo entre generaciones (Parland et al., 2007), índice de conservación genética (Valera et al.,1998), y coancestría y distancias genéticas (Gutiérrez y Goyache, 2005). Los estudios demográficos permiten describir la estructura y dinámica de los individuos en las poblaciones, mientras que los estudios genéticos permiten analizar la variabilidad y evolución del conjunto de genes que constituyen a los individuos (Goyache et al., 2003; Gutiérrez et al., 2003).

La consanguinidad se presenta cuando se aparean animales emparentados. En términos generales, todos los animales de una misma raza están emparentados; sin embargo, se reserva el término de consanguinidad para el apareamiento de animales más emparentados que el promedio de la población (Molina et al., 1995; Bijma et al., 2001; Parland et al., 2007). En las ganaderías de Lidia hay aspectos de manejo y de origen, que influyen en el aumento de los niveles de consanguinidad de los animales. Los aspectos de origen se atribuyen a que los animales proceden de un reducido número de ganaderías fundadoras, mientras que los aspectos de manejo se atribuyen a que la mayoría de las ganaderías producen sus propios reemplazos y el número de sementales es muy reducido. La relación del número de vacas por semental puede ser de 40 ó

Archivos de zootecnia vol. 59, núm. 225, p. 64. 
50 a 1 (Lanfranchi, 1992; Santa-Martina, 2001). Generalmente la consanguinidad se asocia con efectos negativos e indeseables debido a que en algunas especies o razas se ha observado una disminución en el rendimiento medio de algunos caracteres productivos (Burrow, 1993; Falcao et al., 2001; Ruíz-Flores et al., 2006).

En México no se conocen publicaciones científicas que describan y analicen la estructura de los pedigríes y los posibles efectos de la consanguinidad en el comportamiento de los animales de las ganaderías de Lidia. Por lo cual, el objetivo del presente trabajo fue analizar y comparar la estructura y la constitución genética de los pedigríes de cuatro ganaderías mexicanas. Además, se analizó el efecto de la consanguinidad en las notas de tienta y de lidia. Estos resultados son de interés para los criadores, ya que les permite conocer la estructura y constitución genética de sus poblaciones, así como los posibles efectos de la consanguinidad en las notas de comportamiento, para que evalúen y, en su caso, redefinan los criterios de selección y apareamiento entre sus animales.

\section{MATERIAL Y MÉTODOS}

El comportamiento del ganado de Lidia se evalúa por medio de las notas de tienta y de lidia que asigna el ganadero a cada individuo. La nota de tienta hace referencia a la prueba de comportamiento que aplican a los animales a edades tempranas. La nota de lidia analiza el comportamiento de los animales en la plaza de toros. En la tienta y en la lidia se asignan dos evaluaciones, una evaluación para el comportamiento del animal al embestir al caballo y otra para las particularidades de la embestida al torero. Las evaluaciones de tienta y lidia se distribuyen en nueve clases que van desde el muy malo, hasta el superior, asignándole una escala numérica de uno a nueve.

Se analizaron las genealogías y las notas de tienta y de lidia de cuatro ganaderías mexicanas: Los Encinos (ENC), San José (SJO), Fernando de la Mora (FMO) y Montecristo (MCR). Estas ganaderías se ubican en el centro del país (tabla I), forman parte de la Asociación Nacional de Criadores de Toros de Lidia y la información genealógica analizada corresponde al Re-

Tabla I. Ubicación, y descripción de la información genealógica y de comportamiento analizada para las ganaderías evaluadas. (Location of ranches evaluated and description of genealogical and behavioral information analyzed).

\begin{tabular}{|c|c|c|c|c|c|}
\hline Ganadería (Ubicación) & Tienta a caballo & $\begin{array}{l}\text { racterísticas } \\
\text { Tienta a pie }\end{array}$ & $\begin{array}{l}\text { evaluadas } 1,2 \\
\text { Lidia a caballo }\end{array}$ & Lidia a pie & Pedigrí $^{3}$ \\
\hline Los Encinos & 1867 & 1168 & 536 & 558 & 5254 \\
\hline (Pedro Escobedo, Querétaro) & $5,1 \pm 1,9$ & $5,3 \pm 2,0$ & $5,4 \pm 1,7$ & $4,9 \pm 1,9$ & $1904-2006$ \\
\hline Montecristo & 2776 & 2125 & 567 & 1082 & 8279 \\
\hline (Hueyotlipan, Tlaxcala) & $4,0 \pm 1,6$ & $4,6 \pm 1,8$ & $4,7 \pm 1,2$ & $4,5 \pm 1,8$ & $1904-2006$ \\
\hline Fernando de la Mora & 1019 & 921 & 145 & 218 & 3246 \\
\hline (Tecozautla, Hidalgo) & $5,6 \pm 1,7$ & $6,0 \pm 1,8$ & $6,0 \pm 1,6$ & $5,9 \pm 1,9$ & $1938-2002$ \\
\hline San José & 844 & 504 & 202 & 203 & 5875 \\
\hline (Aculco, Edo. de México) & $4,5 \pm 1,7$ & $4,8 \pm 1,8$ & $4,8 \pm 1,7$ & $4,5 \pm 1,6$ & $1904-2002$ \\
\hline
\end{tabular}

${ }^{1}$ Arriba: número de observaciones; parte inferior, media y desviación estándar (las notas de lidia sólo incluyen machos). ²Períodos de evaluación: 1994 a 2006, 1978 a 2006, 1995 a 2002 y 1995 a 2002 , para las ganaderías Los Encinos, Montecristo, Fernando de la Mora y San José, respectivamente. ${ }^{3}$ Parte superior, número de animales; parte inferior, período de año de nacimiento de los animales.

Archivos de zootecnia vol. 59, núm. 225, p. 65. 
gistro Genealógico de Pureza de Raza y Edad del Toro de Lidia. La información genealógica se remonta a 1904, mientras que la información de comportamiento se origina a partir de 1978; en la tabla I se describe la información analizada. Información más específica y a detalle de la estructura y formación de las ganaderías analizadas, fue publicada por Lanfranchi (1992) y Castillo (2003).

Los cálculos de los coeficientes de consanguinidad de cada individuo se realizaron con la subrutina MTDFNRM del programa MTDFREML (Boldman et al., 1995). El análisis de los niveles de consanguinidad a través del tiempo se realizó con diversas subrutinas de SAS (SAS, 2005). Las estimaciones de población base, tamaño efectivo de la población base, consanguinidad promedio esperada, número de animales en la población de referencia, número efectivo de ancestros, aportaciones porcentuales e intervalos generacionales se obtuvieron con el programa ENDOG 3.0 (Gutiérrez y Goyache, 2005). Se consideró como población base a aquellos individuos con al menos un padre desconocido, y como población de referencia a los individuos nacidos en los últimos 7,5 años (equivalentes aproximadamente a una generación).

El número efectivo de ancestros se define como el número de ascendientes, fundadores o no, necesarios para explicar la constitución genética total de la población. La estimación de este parámetro tiene en cuenta que la contribución de los reproductores puede estar desequilibrada y considera, asimismo, los posibles cuellos de botella que haya podido experimentar la población estudiada debido a un excesivo uso de algunos reproductores. El tamaño efectivo de la población base, es el número de animales con padres desconocidos y estima el número de fundadores necesarios para explicar la constitución total de la población, suponiendo que todos ellos realizaran la misma contribución (Boichard et al., 1997). El intervalo generacional se estimó como la edad media de los progenitores cuando nace su progenie seleccionada, para las cuatro vías: padre-hijo, padre-hija, madre-hijo y madrehija (Gutiérrez et al., 2003).

Los análisis del efecto de la consanguinidad sobre las notas de tienta y de lidia se realizaron con el programa MTDFREML. Las notas se analizaron de manera independiente dentro de cada ganadería con un modelo animal que consideró los efectos fijos de grupo contemporáneo (año-época de nacimiento-sexo, el efecto de sexo sólo en tienta), más las covariables lineales de edad y consanguinidad del individuo. Los efectos aleatorios incluidos fueron el efecto genético directo y el error.

\section{RESULTADOSYDISCUSIÓN}

Los niveles de consanguinidad estimados en las ganaderías de Lidia se presentan en la tabla II. Los valores medios estimados en la población total para cada una de las cuatro ganaderías oscilaron entre el 2,4\% y el 12,9\%. Los estimadores máximos por individuo fueron del 47,7\% en la ganadería MCR y alrededor del $40 \%$ en el resto de las ganaderías. Con relación a los animales consanguíneos, la ganadería MCR presentó los valores más altos con el 82,5\% de animales consanguíneos y un promedio de consanguinidad de éstos del 15,6\%; la ganadería SJO presentó la menor proporción de animales consanguíneos (41,6\%), pero el promedio de consanguinidad en esos animales fue superior a los obtenidos en las ganaderías ENC y FMO (12,5\% vs. 8,8\% y 3,9\%, respectivamente). La ganadería MCR presentó los mayores valores de consanguinidad, ya que alrededor del 62,1\% de la población presentó niveles de consanguinidad entre el 10\% y $20 \%$ (tabla III). En las ganaderías ENC y FMO la mayor proporción de animales consanguíneos estuvieron entre el 0\% y 10\% de consanguinidad, y en la ganadería SJO la mayor proporción de animales consanguíneos estuvo entre el 0\% y 15\% (tabla III).

En la figura 1 se presentan las tenden-

Archivos de zootecnia vol. 59, núm. 225, p. 66. 
Tabla II. Niveles estimados de consanguinidad en las ganaderías Los Encinos (ENC), Montecristo (MCR), Fernando de la Mora (FMO) y San José (SJO). (Estimated levels of inbreeding on the ranches Los Encinos (ENC), Montecristo (MCR), Fernando de la Mora (FMO) and San José (SJO)).

\begin{tabular}{lcccc}
\hline Característica & ENC & MCR & FMO & S JO \\
\hline Consanguinidad promedio de la población total, \% & 4,71 & 12,91 & 2,44 & 5,21 \\
Consanguinidad individual máxima, \% & 43,10 & 47,70 & 40,80 & 40,60 \\
No de animales consanguíneos & 2791 & 6834 & 1990 & 2445 \\
Porcentaje de animales consanguíneos & 53,12 & 82,54 & 61,30 & 41,62 \\
Consanguinidad promedio de los animales consanguíneos, \% & 8,81 & 15,59 & 3,97 & 12,50 \\
\hline
\end{tabular}

cias de la consanguinidad de animales consanguíneos y el porcentaje de animales consanguíneos a partir de 1980. La pendiente de la consanguinidad en los animales consanguíneos no fue diferente de cero $(p>0,05)$ en las ganaderías FMO y MCR; mientras que en las ganaderías ENC y SJO hubo una reducción significativa $(\mathrm{p}<0,01)$, con valores de $-0,13 \pm 0,02 \mathrm{y}-0,25 \pm 0,02$, respectivamente. Con respecto a la proporción de animales consanguíneos, en las ganaderías ENC y SJO las pendientes no fueron diferentes de cero ( $\mathrm{p}>0,05)$, contrario a los resultados en las ganaderías FMO y MCR

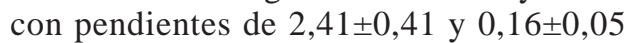
$(\mathrm{p}<0,05)$, respectivamente.

El número de ancestros (fundadores o no) que dio origen a la población de referencia representó 12,3\% en la ganadería MCR, mientras que en las ganaderías FMO, ENC y SJO representaron $17,7 \%, 27,8 \%$ y $34,0 \%$, respectivamente; asimismo, el número de ancestros que explican el 50\% del pedigrí fue menor o igual a 13 animales en las cuatro ganaderías evaluadas; el caso extremo fue la ganadería MCR, con tres animales (tabla

Tabla III. Distribución del número de animales (n) y porcentaje (\%) a través de los diferentes niveles de consanguinidad (F) en las ganaderías Los Encinos (ENC), Montecristo (MCR), Fernando de la Mora (FMO) y San José (SJO). (Distribution of the number of animals (n) and percentage (\%) over the different levels of inbreeding $(F)$ on the ranches Los Encinos (ENC), Montecristo (MCR), Fernando de la Mora (FMO) and San José (SJO)).

\begin{tabular}{ccccccccc}
\hline & \multicolumn{2}{c}{ ENC } & \multicolumn{2}{c}{ MCR } & \multicolumn{2}{c}{ FMO } & \multicolumn{2}{c}{ S JO } \\
Niveles de F (\%) & $\mathrm{n}$ & $\%$ & $\mathrm{n}$ & $\%$ & $\mathrm{n}$ & $\%$ & $\mathrm{n}$ & $\%$ \\
\hline$<0,01$ & 2463 & 46,8 & 1445 & 17,4 & 1262 & 38,7 & 3430 & 58,4 \\
$0,1-5,0$ & 753 & 14,3 & 163 & 1,9 & 1584 & 48,8 & 284 & 4,8 \\
$5,1-10,0$ & 1158 & 22,0 & 732 & 8,8 & 238 & 7,3 & 697 & 11,9 \\
$10,1-15,0$ & 556 & 10,6 & 1849 & 22,3 & 75 & 2,3 & 686 & 11,7 \\
$15,1-20,0$ & 174 & 3,3 & 3295 & 39,8 & 24 & 0,7 & 497 & 8,5 \\
$20,1-25,0$ & 82 & 1,6 & 543 & 6,6 & 9 & 0,3 & 176 & 3,0 \\
$25,1-30,0$ & 29 & 0,6 & 148 & 1,8 & 49 & 1,5 & 68 & 1,2 \\
$30,1-35,0$ & 34 & 0,7 & 56 & 0,7 & 0 & 0,0 & 22 & 0,4 \\
$35,1-40,0$ & 3 & 0,1 & 33 & 0,4 & 4 & 0,1 & 10 & 0,2 \\
$40,1-45,0$ & 2 & 0,0 & 13 & 0,2 & 1 & 0,0 & 5 & 0,1 \\
$45,1-50,0$ & 0 & 0,0 & 2 & 0,0 & 0 & 0,0 & 0 & 0,0 \\
Total & 5254 & 100,0 & 8279 & 100,0 & 3246 & 100,0 & 5875 & 100,0 \\
\hline
\end{tabular}

Archivos de zootecnia vol. 59, núm. 225, p. 67. 
IV). Los tres ancestros que explicaron el $50 \%$ del pedigrí de MCR también tuvieron aportaciones porcentuales importantes en los pedigríes de las ganaderías SJO y ENC, explicando el 22,6\% y 20,2\% de la constitución de su pedigrí, respectivamente. Estos resultados indican lo aisladas o cerradas que han estado estas ganaderías y las bases u orígenes en común que existen entre ellas. $\mathrm{Al}$ analizar los ancestros que determinan la constitución del pedigrí en la ganadería FMO, no se encontró relación con los ancestros de las otras tres ganaderías; siendo ésta una ganadería diferente en sus orígenes.

Algunas publicaciones (Lanfranchi, 1992; Castillo, 2003) que describen el desarrollo histórico de la ganadería de lidia mexicana reportan que la genealogía de los ani- males fundadores en las ganaderías ENC, MCR y SJO, se relaciona entre sí a través de unas ganaderías fundadoras comunes; asimismo, se indica que el origen de la ganadería FMO es independiente al de las otras ganaderías evaluadas. Resultados similares a los de este estudio fueron obtenidos por Santa-Martina (2001), quien reportó que el 35\% de los genes de una ganadería de lidia española provenían también únicamente de tres individuos. El hecho de que un reducido número de ancestros determine un alto porcentaje de la constitución genética de la población, indicaría que las poblaciones han sido sujetas a una alta presión de selección por los criadores que tienden a utilizar intensivamente un reducido número de reproductores (Álvarez et al., 2004). Al respecto, la ganadería FMO fue la que tuvo más
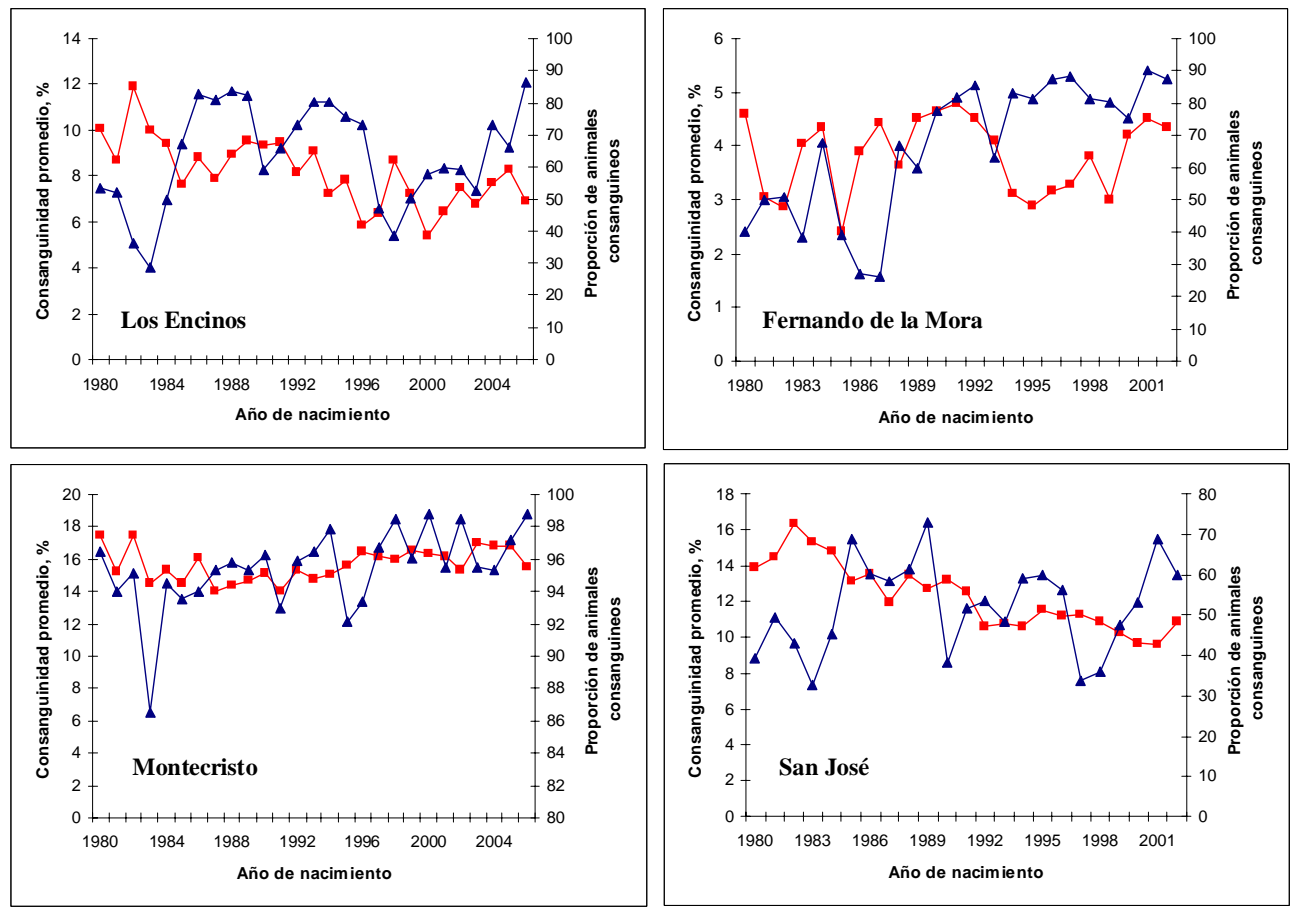

Figura 1. Tendencias de la consanguinidad de animales consanguíneos ( $\square$ ) y porcentaje de animales consanguíneos ( $\Delta$ ) a partir de 1980 en las ganaderías evaluadas. (Trends of inbreeding of inbred animals $(\square)$ and percentage of inbred animals $(\Delta)$ from 1980 in the ranches studied).

Archivos de zootecnia vol. 59, núm. 225, p. 68. 
Tabla IV. Estimación de indicadores poblacionales en cuatro ganaderías de lidia (ENC= Los Encinos; $M C R=$ Montecristo; FMO= Fernando de la Mora; SJO= San José). (Estimation of population indicators on four fighting bull ranches (ENC= Los Encinos; MCR= Montecristo; FMO= Fernando de la Mora; SJO= San José)).

\begin{tabular}{|c|c|c|c|c|}
\hline & \multicolumn{4}{|c|}{ Ganadería } \\
\hline & ENC & MCR & FMO & $\mathrm{SJO}$ \\
\hline Tamaño de la población & 5254 & 8279 & 3246 & 5875 \\
\hline Población base & 840 & 693 & 465 & 1133 \\
\hline Tamaño efectivo de la población base ${ }^{1}$ & 55,5 & 8,7 & 94,6 & 44,5 \\
\hline Consanguinidad promedio esperada ${ }^{2}, \%$ & 0,9 & 5,7 & 0,5 & 1,1 \\
\hline Número de animales en la población de referencia ${ }^{3}$ & 1578 & 1694 & 1399 & 1774 \\
\hline Número de ancestros ${ }^{4}$ & 438 & 208 & 248 & 603 \\
\hline Número de ancestros que explican el $50 \%$ del pedigrí & 13 & 3 & 8 & 11 \\
\hline Número de sementales en el pedigrí & 590 & 748 & 299 & 680 \\
\hline Máximo número de crías de un semental & 156 & 233 & 356 & 170 \\
\hline Promedio del número de crías por semental & 7,7 & 10,2 & 9,4 & 7,0 \\
\hline
\end{tabular}

${ }^{1}$ Individuos con al menos un padre desconocido. ${ }^{2}$ Según el número de ancestros fundadores. ${ }^{3}$ Población de referencia considera a los animales nacidos en los últimos 7,5 años. ${ }^{4} Q u e$ dan origen a la población de referencia.

crías de un único semental (356); sin embargo, la ganadería MCR presentó el promedio más alto $(10,2)$ de crías por semental (tabla IV). De acuerdo con estos resultados, parecería que el excesivo uso de algunos sementales no ha repercutido notoriamente en los niveles de consanguinidad en la ganadería FMO, a diferencia de lo observado en la ganadería MCR. Esto podría indicar que aparte del reducido número de sementales, la ganadería MCR ha estado cerrada a la utilización de reproductores originarios de otras ganaderías, al contrario de lo que ocurre en FMO, que podría haber introducido algunos sementales de otras ganaderías. Con respecto a este planteamiento, Castillo (2003) informó de la importación e introducción de sementales de origen español en las ganaderías SJO y ENC a mediados de la década de los 90.

En la tabla V se presentan los intervalos generacionales para las cuatro ganaderías analizadas. La edad promedio de los sementales y vientres cuando nacieron los machos seleccionados fue mayor que cuan- do nacieron las hembras seleccionadas, lo que indica que la selección de machos se realiza considerando una mayor cantidad de información que para el caso de las hembras. En particular el mayor intervalo madrehijo con respecto al de padre-hijo podría explicarse a que los sementales se utilizan por un menor tiempo en la ganadería. Con excepción del intervalo padre-hija, los mayores intervalos generacionales se obser-

Tabla V. Intervalos generacionales para las ganaderías Los Encinos (ENC), Montecristo (MC), Fernando de la Mora (FMO) y San José (SJO). (Generation intervals for the ranches Los Encinos (ENC), Montecristo (MC), Fernando de la Mora (FMO) and San José (SJO)).

\begin{tabular}{lcccc}
\hline Vía & ENC & MCR & FMO & SJO \\
\hline Padre-hijo & $7,5 \pm 0,2$ & $7,6 \pm 0,1$ & $7,6 \pm 0,2$ & $7,4 \pm 0,2$ \\
Padre-hija & $7,3 \pm 0,2$ & $7,1 \pm 0,1$ & $7,1 \pm 0,2$ & $7,2 \pm 0,2$ \\
Madre-hijo & $7,8 \pm 0,2$ & $8,2 \pm 0,2$ & $8,0 \pm 0,3$ & $7,8 \pm 0,2$ \\
Madre-hija & $7,2 \pm 0,2$ & $7,8 \pm 0,2$ & $7,6 \pm 0,2$ & $7,4 \pm 0,2$ \\
Total & $7,3 \pm 0,1$ & $7,5 \pm 0,0$ & $7,5 \pm 0,1$ & $7,4 \pm 0,1$ \\
\hline
\end{tabular}

Archivos de zootecnia vol. 59, núm. 225, p. 69. 
varon en las ganaderías MCR y FMO, lo que sugiere una mayor presión de selección en estas ganaderías.

Los intervalos generacionales en el presente estudio son superiores a los encontrados en poblaciones de bovinos Charolais, Limousine, Hereford, Angus, Simmental y Holstein por Parland et al. (2007), y a los reportados por Gutiérrez et al. (2003), quienes evaluaron ocho razas de bovinos para carne en España. Las diferencias en los intervalos generacionales de ganado de Lidia con respecto a razas especializadas de bovinos para carne se pueden atribuir a los diferentes esquemas de manejo reproductivo. En ganado de Lidia, los sementales al inicio de su vida reproductiva se utilizan con ciertas reservas, hasta que cierto número de crías expresan comportamientos satisfactorios para el ganadero en las notas de tienta o de lidia, se inicia el uso intensivo de los mismos.

El efecto de edad del animal sólo fue significativo $(p<0,05)$ en las ganaderías ENC y MCR (tabla VI), observándose que a medida que la edad del animal aumentó, la calificación en las notas al caballo se incre- mentó ocurriendo lo contrario para las notas al torero. El efecto de la consanguinidad del animal sólo fue significativo $(\mathrm{p}<0,05)$ en las ganaderías ENC y SJO; en ambas ganaderías la calificación en las notas de tienta aumentó conforme los niveles de consanguinidad del animal fueron mayores, mientras que para la lidia al torero se observó un efecto similar sólo en SJO. La magnitud de los coeficientes de regresión varió entre 0,02 y 0,05 unidades en las notas por unidad porcentual de la consanguinidad; estos valores pueden considerarse de magnitud pequeña, ya que por ejemplo, en un animal con un porcentaje de consanguinidad de 10\% (promedio de la consanguinidad de los animales consanguíneos en las cuatro ganaderías) implicaría aumentos en las notas de entre 0,2 y 0,5 .

La dirección del efecto de consanguinidad de estos análisis difiere a los reportados en ganado de Lidia (Núñez y Castro, 1998), donde se observaron reducciones en bravura, fuerza y nota de tienta (promedio de bravura, estilo, fuerza, recorrido y prontitud) a medida que se incrementó la consanguinidad. Asimismo, en razas de bovi-

Tabla VI. Coeficientes de regresión para las covariables incluidas en los modelos de análisis de las notas de tienta y lidia, por ganadería. (Regression coefficients for the covariables included in the models to analyze testing and fighting notes, by ranch).

\begin{tabular}{|c|c|c|c|c|}
\hline Covariable en el modelo & Tienta a caballo & Tienta a pie & Lidia a caballo & Lidia a pie \\
\hline \multicolumn{5}{|l|}{ Los Encinos } \\
\hline Edad del individuo & $-0,12 \pm 0,12$ & $-0,32 \pm 0,15^{*}$ & $0,21 \pm 0,11^{*}$ & $-0,05 \pm 0,01^{*}$ \\
\hline Consanguinidad del individuo & $0,02 \pm 0,01^{*}$ & $0,03 \pm 0,01^{*}$ & $0,02 \pm 0,02$ & $-0,01 \pm 0,02$ \\
\hline \multicolumn{5}{|l|}{ Montecristo } \\
\hline Edad del individuo & $0,25 \pm 0,01^{*}$ & $-0,14 \pm 0,12$ & $0,28 \pm 0,08^{*}$ & $-0,01 \pm 0,08$ \\
\hline Consanguinidad del individuo & $-0,01 \pm 0,01$ & $0,02 \pm 0,01$ & $-0,02 \pm 0,01$ & $0,02 \pm 0,02$ \\
\hline \multicolumn{5}{|l|}{ Fernando de la Mora } \\
\hline Edad del individuo & $0,27 \pm 0,19$ & $0,19 \pm 0,21$ & $-0,48 \pm 0,26$ & $0,19 \pm 0,26$ \\
\hline Consanguinidad del individuo & $0,01 \pm 0,01$ & $0,01 \pm 0,01$ & $0,05 \pm 0,04$ & $0,04 \pm 0,04$ \\
\hline \multicolumn{5}{|l|}{ San José } \\
\hline Edad del individuo & $-0,32 \pm 0,19$ & $0,07 \pm 0,24$ & $-0,20 \pm 0,20$ & $0,14 \pm 0,16$ \\
\hline Consanguinidad del individuo & $0,03 \pm 0,01^{*}$ & $0,04 \pm 0,01^{*}$ & $0,02 \pm 0,03$ & $0,05 \pm 0,02^{*}$ \\
\hline
\end{tabular}

*Coeficientes de regresión significativos $(p<0,05)$.

Archivos de zootecnia vol. 59, núm. 225, p. 70. 
nos para carne y leche se ha observado disminución en el nivel productivo cuando se incrementa la consanguinidad (Burrow, 1993; Falcao et al., 2001; Ruíz-Flores et al., 2006). Los resultados del presente trabajo contrastan con los efectos de depresión endogámica comúnmente observados en caracteres cuantitativos o poligénicos (Falconer y Mackay, 1996). Las pendientes positivas observadas de la consanguinidad en las notas de tienta y lidia al torero, se pudieran atribuir a que los criadores a través de los años han practicado apareamientos endogámicos buscando fijar características deseables y seleccionando reproductores sobresalientes; lo anterior pudo provocar que los animales contemporáneos con mejores notas de tienta tengan parentescos elevados entre ellos. Considerando las discrepancias de los resultados de este trabajo con los esperados, es conveniente realizar otros estudios para evaluar el efecto de la consanguinidad en ganado de Lidia con mayor precisión.

\section{CONCLUSIONES}

La proporción de animales consanguí-

\section{BIBLIOGRAFÍA}

Álvarez S., A., Gutiérrez, J.P., Fernández, I., Royo, L.J., Álvarez, I., Gómez, E. y Goyache, F. 2004. Conservación de la oveja Xalda de Asturias. Anim. Gen. Res. Inf., 34: 41-49.

Bijma, P., Van Arendonk, J.A.M. and Woolliams, J.A. 2001. Predicting rates of inbreeding for livestock schemes. J. Anim. Sci., 79: 840853

Boichard, D., Maignel, L. and Verrier, É. 1997. The value of using probabilities of gene origin to measure genetic variability in a population. Genet. Sel. Evol., 29: 5-23.

Boldman, K.G., Kriese, L.A., Van Vleck, L.D., Van Tassell, C.P. and Kachman, S.D. 1995. A Manual for use of MTDFREML. A set of programs to obtain estimates of variances and covariances (Draft). USDA. ARS. 114 pp.

Burrow, H.M. 1993. The effects of inbreeding in neos y los niveles de consanguinidad fueron de medianos a altos en las ganaderías Los Encinos, Fernando de la Mora, y San José, mientras que en Montecristo fueron los más altos.

El número de ancestros (fundadores o no) que explican la constitución del pedigrí es reducido en las 4 ganaderías analizadas. Las ganaderías Montecristo, San José, y Los Encinos tienen un origen común en las genealogías de los animales fundadores.

Se ha detectado una asociación significativa entre el nivel de consanguinidad del animal y las notas de tienta de las ganaderías Los Encinos y San José, y la nota de lidia al torero en esta última ganadería. Considerando las discrepancias de los resultados de este trabajo con los esperados, se recomienda continuar el estudio del efecto de la consanguinidad en ganado de Lidia.

\section{AGRADECIMIENTOS}

Se agradece a los propietarios de las ganaderías evaluadas el proporcionar la información genealógica y de comportamiento correspondiente para el desarrollo del presente estudio.

beef cattle. Anim. Breed. Abstr., 65: 477-495. Castillo G., E. 2003. Nuestro toro. Asociacion Nacional de Criadores de Toros de Lidia. México, D.F. 483 pp.

Falcao, S., Filho M., R., Magnabosco U., C., Bozzi, R. e Lima M., F.A. 2001. Efeitos da endogamia sobre características de reproducao, crescimento e valores genéticos aditivos de bovinos de raca Pardo-Suíça. Rev. Bras. Zootecn., 30: 83-92.

Falconer, D.S. and Mackay, T.F.C. 1996. An Introduction to quantitative genetics. $4^{\text {th }}$ ed. Longman Pub. London, UK. 464 pp.

Goyache, F., Gutiérrez, J.P., Fernández, I., Gómez, E., Álvarez, I., Diez, J. and Royo, L.J. 2003. Using pedigree information to monitor genetic variability of endangered populations: the Xalpa sheep breed of Asturias as an example. J.

Archivos de zootecnia vol. 59, núm. 225, p. 71. 


\section{DOMÍNGUEZ VIVEROSETAL.}

Anim. Breed. Genet., 120: 95-105.

Gutiérrez, J.P., Altarriba, J., Díaz, C., Quintanilla, R., Cañon, J. and Piedrafita, J. 2003. Pedigree análisis of eight Spanish beef cattle breeds. Genet. Sel. Evol., 35: 43-63.

Gutiérrez, J.P. and Goyache, F. 2005. A note on ENDOG: a computer program for analysis pedigree information. J. Anim. Breed. Genet, 122: $172-176$

Lanfranchi, H. 1992. Historia del Toro Bravo Mexicano. Asociación Nacional de Criadores de Toros de Lidia. México, D.F. 401 pp.

McParland, S., Kearney, J.F., Rath, M. and Berry, D.P. 2007. Inbreeding trends and pedigree analysis of Irish dairy and beef cattle populations. J. Anim. Sci., 85: 322-331.

Molina, A., Rodero, A. y Valera, M. 1995. Análisis genético de los niveles de consanguinidad en la raza Retinta. Arch. Zootec., 44: 257-265.

Núñez D., R. y Castro G., H. 1998. Bases para la evaluación de los recursos genéticos de la ganadería de Lidia en México. En: Memorias del tercer foro de análisis de los recursos genéti- cos: ganadería ovina, caprina, porcina, avícola apícola, equina y de Lidia. México, D.F. 27 y 28 de agosto de 1998. pp. 223-230.

Santa-Martina M., J. 2001. El toro de Lidia: conservación de los recursos genéticos. Arch. Zootec., 50: 35-40.

Ruíz-Flores, A., Núñez-Domínguez, R., RamírezValverde, R., Domínguez-Viveros, J., MendozaDomínguez, M. y Martínez-Cuevas, E. 2006. Niveles y efectos de la consanguinidad en variables de crecimiento y reproductivas en bovinos Tropicarne y Suizo. Agrociencia, 40: 289-301.

SAS. 2005. SAS/STAT User's Guide. (Release 9.0). SAS Inst. Inc. Cary. NC. USA.

Valera, M., Molina, A. y Rodero, A. 1998. Índice de conservación genética en la estirpe Cartujana del caballo Pura Sangre Española. Arch. Zootec., 47: 180-198.

Vasallo, J.M., Díaz, C. and García M., J.R. 1986. A note on the population structure of the Avileña breed of cattle in Spain. Livest. Prod. Sci., 15: 285-288.

Archivos de zootecnia vol. 59, núm. 225, p. 72. 